\title{
Expression of the Ets-1 Proto-Oncogene in Melanocytic Lesions
}

\author{
Connie A. Keehn, M.D., Bruce R. Smoller, M.D., Michael B. Morgan, M.D. \\ Department of Pathology at the University of South Florida College of Medicine (CAK, MBM); \\ Dermatopathology Division, University of Arkansas for Medical Sciences (BRS); and Department of \\ Pathology, James A. Haley Veteran's Hospital (MBM)
}

Ets-1 oncoprotein is a transcription factor known to regulate the expression of numerous genes important in extracellular matrix remodeling and angiogenesis. Up-regulation of Ets-1 has been shown to be important in a variety of human malignancies and to correlate with prognosis. To our knowledge, this oncoprotein has not been examined in melanocytic lesions. A series of 10 cutaneous melanomas and 24 benign melanocytic lesions with patient records were independently examined for diagnosis confirmation and immunohistochemical expression by two dermatopathologists. The immunohistochemical expression for Ets-1 (Novocastra, Newcastle upon Tyne, $\mathrm{UK}$ ) was scored by an average of the mean labeling intensity; no nuclear staining = 0 , weak nuclear staining $=1$, moderate $=2$, and intense $=3$. Ets-1 expression was statistically assessed by the one-way analysis of variance (ANOVA) comparing the mean labeling intensity of melanoma to benign melanocytic nevi. All of the benign melanocytic lesions exhibited negative to weak nuclear staining, with an average mean labeling intensity of 0.4. Melanoma in situ exhibited moderate nuclear staining, for a mean labeling intensity of 2.0, whereas all conventional invasive melanomas exhibited moderate to strong nuclear staining, with a mean labeling intensity of 2.7. Metastatic melanoma exhibited very strong nuclear staining, with a mean labeling intensity of 3.0. Invasive desmoplastic melanoma, like melanoma in situ, showed moderate nuclear staining with a mean labeling intensity of 2.1. There was a trend toward more intense staining with melanoma progression. A statistically significant difference in the mean labeling intensity of Ets-1 was seen between invasive melanoma and

\footnotetext{
Copyright () 2003 by The United States and Canadian Academy of Pathology, Inc.

VOL. 16, NO. 8, P. 772, 2003 Printed in the U.S.A

Date of acceptance: May 5, 2003.

Address reprint requests to: Connie A. Keehn, M.D., 15350 Amberly Drive

\#1714, Tampa, FL 33647; fax: 813-903-4813; e-mail: ckeehn@hsc.usf.edu.

DOI: 10.1097/01.MP.0000082395.59356.4F
}

benign melanocytic nevi $(P<.0001)$. Ets-1 oncoprotein expression, however, does not distinguish among benign melanocytic lesions. Staining intensity and pattern might be a useful adjunct with histomorphology in distinguishing invasive melanoma from benign melanocytic nevi. Furthermore, Ets-1 expression may be an important pathogenic mechanism and predictor of aggressive biologic behavior of cutaneous melanoma, with a trend toward staining intensity increasing as Clark stage increases.

KEY WORDS: Ets-1, Immunohistochemistry, Melanocytic nevi, Melanoma.

Mod Pathol 2003;16(8):772-777

The Ets-1 oncoprotein is a transcription factor known to regulate the expression of numerous genes important in cell proliferation and differentiation (1). It is implicated in cell migration and tumor invasion by inducing the expression of several genes encoding matrix-degrading proteases, including urokinase-type plasminogen activator, stromelysin, and collagenase (2-6). Ets-1 also promotes angiogenesis by activating matrix metalloproteinases in endothelial cells $(2,5-7)$.

Up-regulation of Ets-1 has been shown to be important in carcinogenesis and/or tumor progression in a variety of human malignancies, including carcinomas of the stomach $(8,9)$, pancreas $(10)$, bile ducts (11), colon (12-14), liver $(15,16)$, esophagus (17), oral cavity $(18-20)$, lung $(21,22)$, thyroid (23, $24)$, and ovary $(25,26)$, as well as in malignant meningiomas (27), malignant astroglial tumors (28), and angiosarcoma of the skin (29). To our knowledge, this oncoprotein has not been examined in melanocytic lesions.

Molecular markers important in the pathogenesis and prognosis of melanoma are an active area of current research. These include important downstream effectors of Ets-1 regulation including urokinase-type plasminogen activator, stromelysin, and collagenase $(30,31)$. Known prognostic indica- 
tors in cutaneous melanoma include the Breslow microstage, followed by the Clark level of invasion, growth phase, tumor mitotic rate, the presence of host tumor-infiltrating lymphocytes within the vertical growth phase, presence or absence of histologic regression, and ulceration. However, these indicators cannot precisely predict the clinical course of this disease for the individual patient. New molecular prognostic markers may be useful to provide for more accurate diagnosis and prognosis. In addition, such markers may help improve our understanding of the biology of melanoma and potentially offer new targets for novel treatment strategies. We studied a series of benign and malignant melanocytic lesions for the immunohistochemical expression of Ets-1 in order to discern a possible difference in expression between benign and malignant lesions and to evaluate the relationship between intensity of staining and increasing tumor depth in melanoma.

\section{MATERIALS AND METHODS}

\section{Cases and Tissues}

We studied a series of 10 cutaneous melanomas and 24 benign melanocytic lesions. The cutaneous melanomas consisted of 2 melanomas in situ, 2 Clark Stage II melanomas, 1 Clark Stage III melanoma, 1 Clark Stage IV melanoma, 2 metastatic melanomas, and 2 desmoplastic melanomas. The benign melanocytic lesions consisted of 5 Spitz compound nevi, 6 dysplastic common nevi with moderate atypia, 6 common blue nevi, 4 intradermal nevi, and 3 congenital compound nevi. All cases along with patient records were independently examined for diagnosis confirmation by two dermatopathologists (MM, BS).

\section{Immunohistochemistry}

Formalin-fixed and paraffin-embedded tissues were cut into $3-\mu \mathrm{m}$ sections and dried overnight at room temperature. Sections were deparaffinized in xylene and rehydrated through descending grades of ethanol to deionized water. High-temperature antigen unmasking was performed in a microwave using $10 \mathrm{~mm}$ citrate buffer, $\mathrm{pH}$ 6.0. Slides were cooled, washed in deionized water, and placed in TBS/Tween for 5 minutes. Deparaffinized sections were stained on the DAKO Autostainer using the DAKO LSAB + Peroxidase detection kit (DAKO Corporation, Carpinteria, CA). All incubations were performed at room temperature $\left(25^{\circ} \mathrm{C}\right)$. Endogenous peroxidase was blocked with $3 \%$ aqueous hydrogen peroxide, and endogenous biotin was blocked with the Vector Avidin/Biotin blocking kit (Vector Laboratories, Burlingame, CA). Sections were preincubated with DAKO Protein Block (surefire) to prevent nonspecific binding. A mouse monoclonal antibody against human Ets-1 (prokaryotic recombinant protein corresponding to amino acids 226 to 454 of the human Ets-1 oncoprotein; Novocastra Laboratories Ltd, Newcastle upon Tyne, UK via Vector Laboratories, Burlingame, CA) was used at a dilution of 1:50 for 60 minutes. The slides were sequentially incubated with biotinylated anti-mouse, -rabbit, -goat IgG, and streptavidin peroxidase and reaction products were visualized with the chromogenic substrate, NovaRed (Vector Laboratories, Burlingame, CA). Slides were removed from the Autostainer, counterstained with modified Mayer's hematoxylin, dehydrated, cleared, and mounted with resinous mounting medium. Mouse IgG was used as the negative control serum. Tonsil served as the positive control tissue. Lymphocytes served as the internal positive control. Analysis of the immunohistochemical staining was performed by two dermatopathologists (MM, BS). In each case, 100 consecutive melanocytic nuclei were evaluated for nuclear staining intensity at $400 \times$ magnification in nonoverlapping fields. An average score was reported for each slide. Ets-1 expression was scored by an average of the mean labeling intensity; no nuclear staining of tumor cells $=0$, weak nuclear staining $=1$, moderate nuclear staining $=2$, and intense nuclear staining $=3$.

\section{Statistical Analysis}

Ets-1 expression was statistically assessed by the one-way analysis of variance (ANOVA), comparing the mean labeling intensity of melanoma to benign melanocytic nevi.

\section{RESULTS}

The positive control tissue (tonsil) showed intense nuclear staining of lymphocytes. Slides incubated with negative control serum exhibited no tonsillar cellular staining. The skin specimens showed weak to moderate nuclear staining of the basilar keratinocytes, with weak nuclear staining of the granular layer. Intraepidermal melanocytes showed no nuclear staining. Within the dermis, endothelial cells showed moderate nuclear staining. Dermal lymphocytes exhibited intense nuclear staining. Dermal fibrocytes and subcutaneous fat adipocytes were negative. Weak background cytoplasmic staining was seen within keratinocytes.

\section{Immunopositivity of Ets-1 in Melanoma and Benign Melanocytic Lesions}

All of the benign melanocytic lesions studied (Spitz compound nevi, dysplastic common nevi, 
common blue nevi, intradermal nevi, and congenital compound nevi) exhibited negative to weak nuclear staining, for an average mean labeling intensity of 0.4. Melanoma in situ exhibited moderate nuclear staining, for an average mean labeling intensity of 2.0. All conventional invasive melanomas exhibited moderate to strong nuclear staining, with an average mean labeling intensity of 2.7. Metastatic melanoma exhibited very strong nuclear staining, with an average mean labeling intensity of 3.0. Invasive desmoplastic melanoma, like melanoma in situ, showed moderate nuclear staining with an average mean labeling intensity of 2.1. All lesions exhibited diffuse staining patterns, with the intensity of staining similar throughout a given lesion. A statistically significant difference in the mean labeling intensity of Ets-1 expression was seen between invasive melanoma and benign melanocytic nevi $(P<.0001)$. Ets- 1 oncoprotein expression, however, did not distinguish among the different benign melanocytic lesions. (See Fig. 1)

\section{Relation between Ets-1 Expression and Clark Stage in Melanoma}

There was a trend of increasing intensity of Ets-1 expression with increasing Clark stage among conventional invasive melanomas. (See Figs. 2-6)

\section{DISCUSSION}

ETS- 1 is a proto-oncogene that is part of a family of proteins that play a central role in cellular differ-

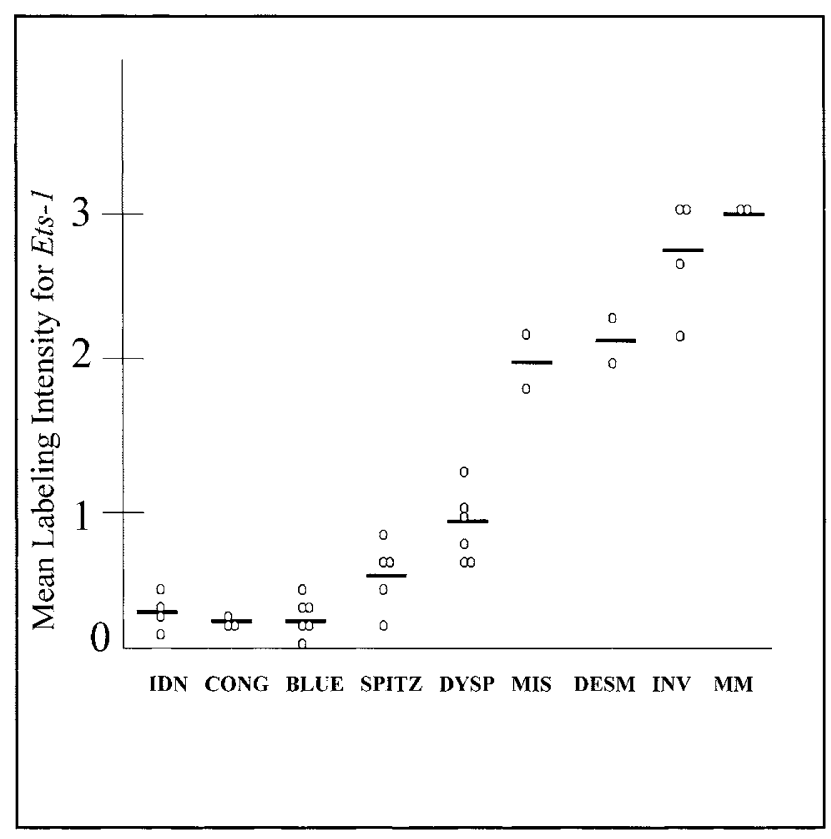

FIGURE 1. Mean labeling intensity for Ets-1 expression in melanocytic lesions. IDN $=$ intradermal nevi, $\mathrm{CONG}=$ congenital compound nevi, BLUE = common blue nevi, SPITZ $=$ Spitz compound nevi, DYSP $=$ dysplastic common nevi with moderate atypia, MIS = melanomas in situ, DESM = desmoplastic melanoma, INV = conventional invasive melanomas, $\mathrm{MM}=$ malignant melanomas.

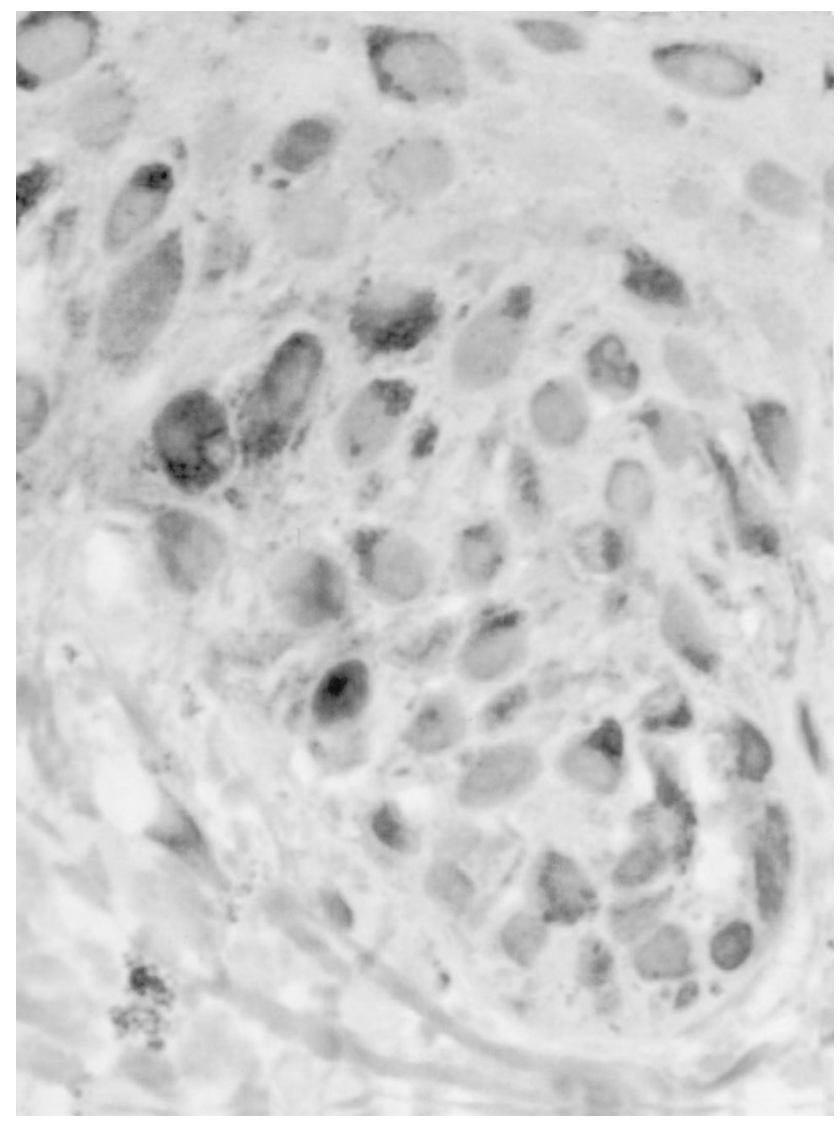

FIGURE 2. Ets-1 expression for melanoma in situ.

entiation, activation, and proliferation. Ets-1 is specifically involved in lymphoid differentiation, as well as being expressed in adult lung, gut mesenchymal tissue, and bone marrow (32). It is also normally expressed within the skin and in proliferating endothelial cells. Chromosomal translocations involving the Ets gene are pathologically associated with some types of lymphoma and soft tissue neoplasms (33). Markedly elevated Ets-1 expression has been demonstrated within endothelial cells in angiosarcomas but is not seen in benign endothelial proliferations such as hemangiomas and pyogenic granulomas (29). It has been suggested that Ets-1 promotes angiogenesis by inducing expression of metalloproteinases and integrin B3 $(7,29)$. It has also been suggested that Ets-1 may prevent apoptosis, but those same investigators found that endothelial cells demonstrated more apoptosis in the presence of Ets-1 (7). It has also been shown that mutations in MMP-1 may result in additional Ets-1 binding sites and in increased transcription of these proteins. In cell culture, melanoma cells demonstrated these mutations (34).

In the present study, we demonstrated strong nuclear staining with Ets-1 in malignant melanocytes but negative to weak nuclear staining in a variety of benign melanocytic lesions. Further, the intensity of expression of Ets-1 increased with in- 


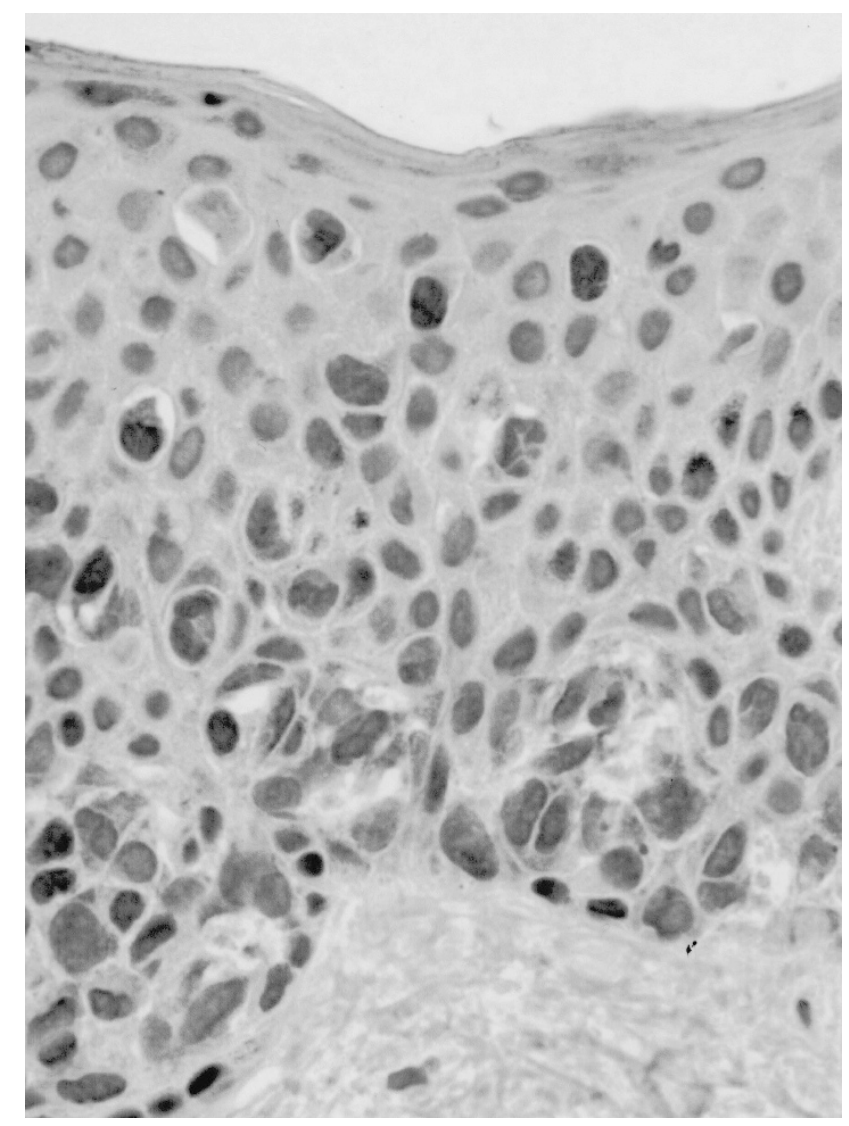

FIGURE 3. Ets-1 expression for Clark Stage II melanoma.

creasing Clark stage in the cases studied. Although increased expression was seen within the intraepidermal melanocytes of melanoma in situ, the expression was more intense within deeply invasive tumors. Expression of MMP-1 has been shown to be up-regulated within dermal fibroblasts of deeply invasive melanomas (35). It can be speculated based on cell culture data (34) that the increase in MMP-1 expression is possibly related to Ets-1 expression by the melanoma cells. It follows that increased metastatic potential and aggressive behavior in these lesions may be directly tied into the progressive capacity for melanoma cells to express Ets-1.

The ability of Ets-1 to induce endothelial cell proliferation and activation, resulting in angiogenesis, provides another potential mechanism of action for this proto-oncogene. The correlation between angiogenesis and aggressive behavior and poor prognosis has been well documented for melanomas (36-40). It is possible that up-regulation of Ets-1 by invasive melanoma cells documented in our study could facilitate access of melanoma to dermal vasculature and ultimate metastasis.

Ets-1 over-expression was not seen in a variety of benign melanocytic lesions. The differential expression of other proteins such as HMB-45, MART-1, and MIB-1, has been touted as a good discriminator

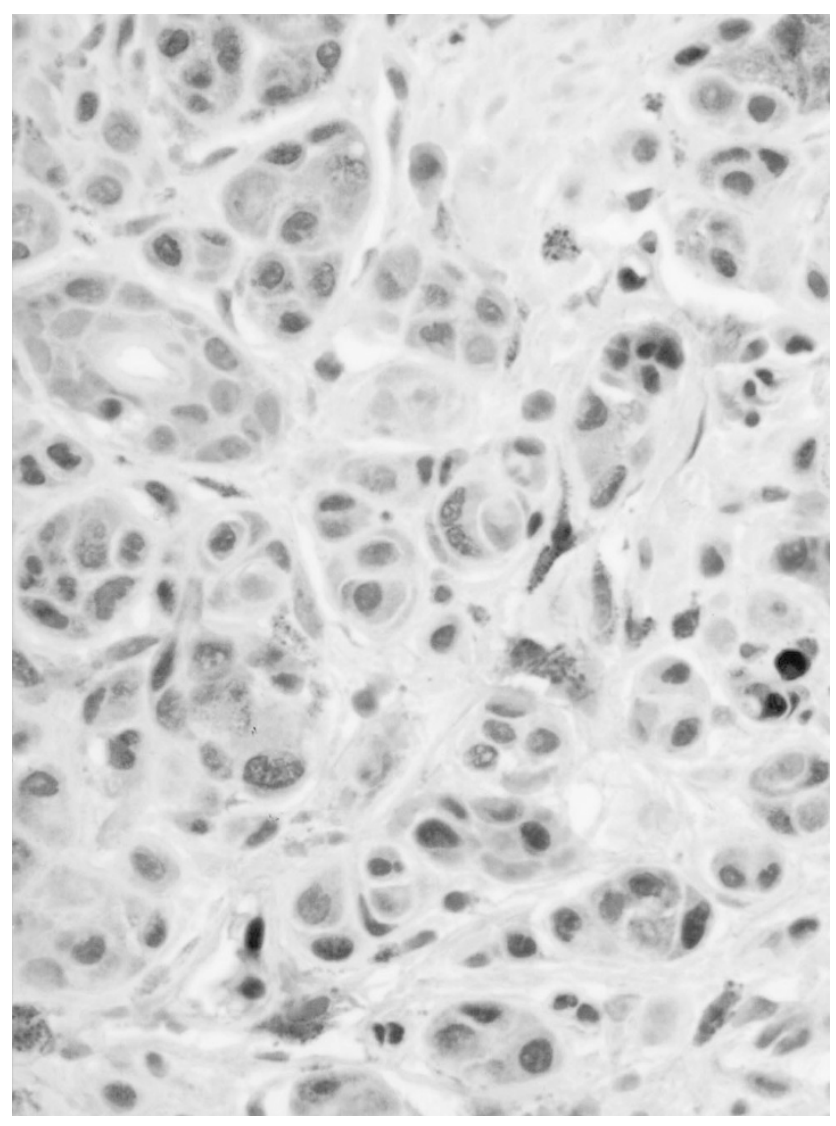

FIGURE 4. Ets-1 expression for Clark Stage III melanoma.

of benign and malignant melanocytic proliferations $(41,42)$. However, in most of these situations, junctional melanocytes express these proteins in a similar manner regardless of their ultimate biologic behavior. Similarly, dermal melanocytes within Spitz's nevi, cellular blue nevi, dysplastic nevi, and other benign lesions have also been shown to overexpress these other proteins. These observations have relegated these markers to a limited role in discriminating among melanocytic nevi and melanoma. In the current study, we demonstrated negative to weak staining in all of our benign melanocytic proliferations, in contrast to the relatively strong nuclear staining in the vast majority of our melanomas. Although based upon a relatively small number of cases, our preliminary data suggest that overexpression of Ets-1 may be a useful means for discriminating invasive melanoma from melanocytic nevi.

In summary, we have demonstrated that Ets-1 is overexpressed within malignant melanoma cells, but similar overexpression is not seen within benign melanocytic proliferations. Our preliminary data suggest that Ets-1 expression may be useful in helping to discriminate benign from malignant melanomas in difficult cases; however, this statement is made with caution, and additional studies are necessary to examine a more complete range of 


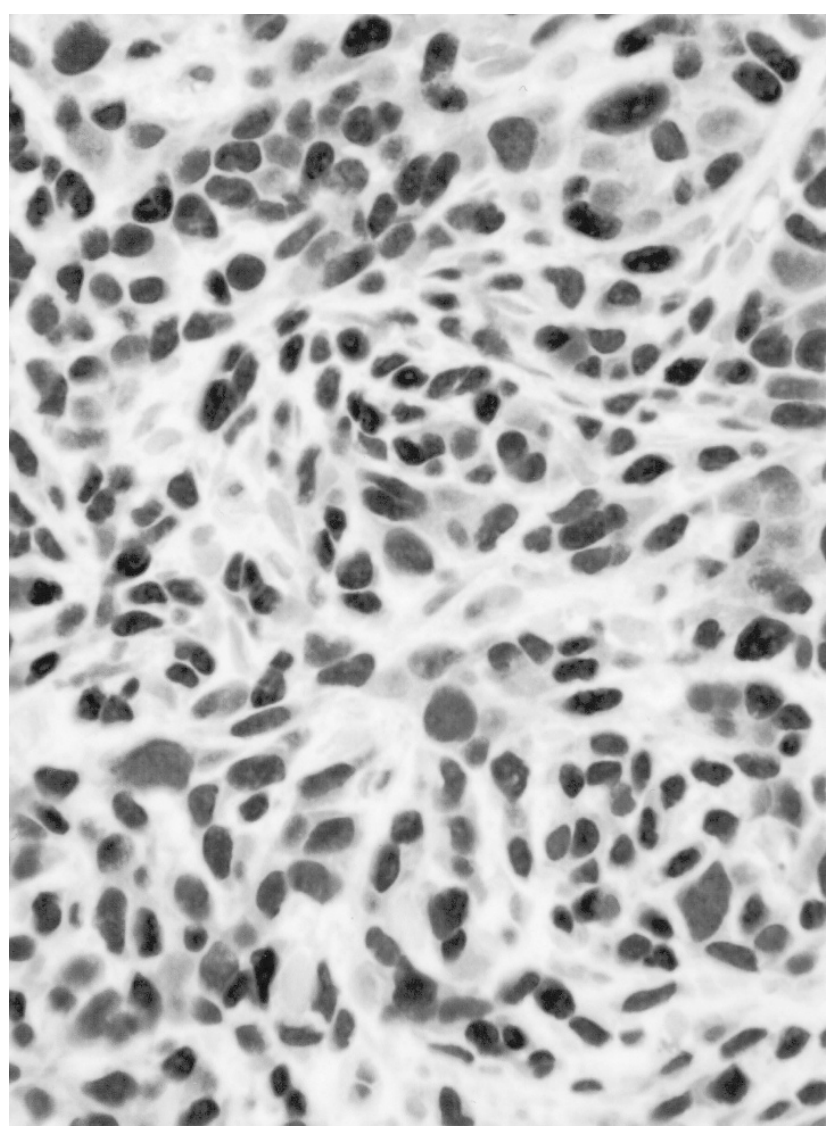

FIGURE 5. Ets- 1 expression for Clark Stage IV melanoma.

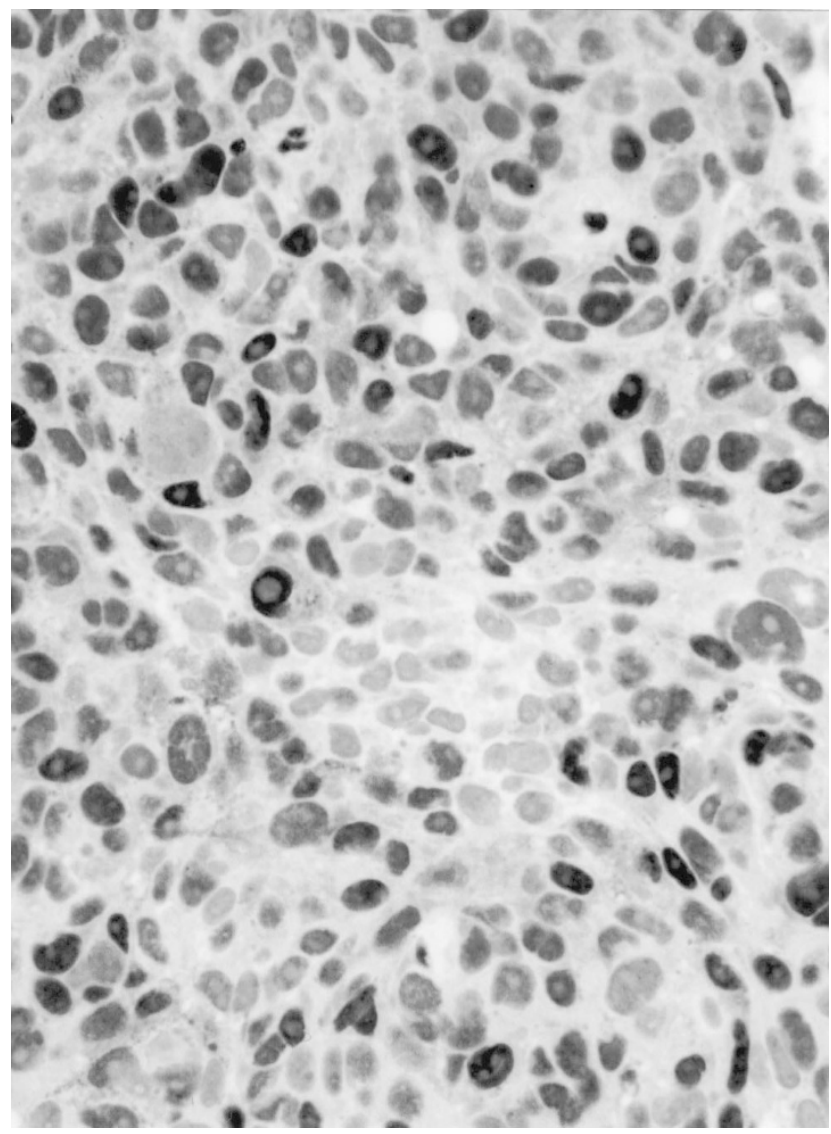

FIGURE 6. Ets-1 expression for metastatic melanoma. melanocytic proliferations and Ets-1 expression. In addition, Ets-1 expression by melanoma cells may explain the previously observed neovascularization underlying these lesions and the increased MMP-1 expression seen underlying highly aggressive tumors. Additional studies examining these relationships may result in potential therapeutic interventions and a better understanding of the pathogenesis of metastasis in primary cutaneous melanomas.

\section{REFERENCES}

1. Lewin B. Oncogenic conversion by regulatory changes in transcription factors. Cell 1991;64:303-12.

2. Vandenbunder B, Wernert N, Queva C, Desbiens X, Stehelin D. Does the transcription factor c-ets1 take part in the regulation of angiogenesis and tumor invasion? Folia Biol 1994; 40:301-13.

3. Liotta LA, Stetler-Stevenson WG. Metalloproteinases and cancer invasion. Semin Cancer Biol 1990;1:99-106.

4. Nakayama T, Ito M, Ohtsuru A, Naito S, Sekine I. Expression of the ets-1 proto-oncogene in human colorectal carcinoma. Mod Pathol 2001;14:415-22.

5. Naito S, Shimizu S, Matsuu M, Nakashima M, Nakayama T, Yamashita S, et al. Ets-1 upregulates matrix metalloproteinase-1 expression through extracellular matrix adhesion in vascular endothelial cells. Biochem Biophys Res Commun 2002;291:130-8.

6. Behrens P, Rothe M, Wellmann A, Krischler J, Wernert N. The Ets-1 transcription factor is up-regulated together with MMP 1 and MMP 9 in the stroma of pre-invasive breast cancer. J Pathol 2001;194:43-50.

7. Sato Y, Teruyama K, Nakano T, Oda N, Abe M, Tanaka K, et $a l$. Role of transcription factors in angiogenesis: Ets- 1 promotes angiogenesis as well as endothelial apoptosis. Ann $\mathrm{N}$ Y Acad Sci 2001;947:117-23.

8. Nakayama T, Ito M, Ohtsuru A, Naito S, Nakashima M, Fagin JA, et al. Expression of Ets-1 proto-oncogene in human gastric carcinoma: correlation with tumor invasion. Am J Pathol 1996;149:1931-9.

9. Tsutsumi S, Kuwano H, Asao T, Nagashima K, Shimura T, Mochiki E. Expression of the Ets-1 angiogenesis-related protein in gastric cancer. Cancer Lett 2000;160:45-50.

10. Ito T, Nakayama T, Ito M, Naito S, Kanematsu T, Sekine I. Expression of ets-1 proto-oncogene in human pancreatic carcinoma. Mod Pathol 1998;11:209-15.

11. Ito Y, Miyoshi E, Takeda T, Sakon M, Tsujimoto M, Yokosaki $\mathrm{Y}$, et al. ets-1 expression in extrahepatic bile duct carcinoma and cholangiocellular carcinoma. Oncology 2000;58:248-52.

12. Ito $\mathrm{Y}$, Takeda T, Okada M, Matsuura N. Expression of ets-1 and ets-2 in colonic neoplasms. Anticancer Res 2002;22: 1581-4.

13. Sato T, Miwa A. Ets-1 and integrin beta3 for lung metastasis from colorectal cancer. APMIS 2002;110:347-53.

14. Nakayama T, Ito M, Ohtsuru A, Naito S, Sekine I. Expression of the ets-1 proto-oncogene in human colorectal carcinoma. Mod Pathol 2001;14:415-22.

15. Kanda K, Nakayama T, Onizuka S, Tomioka T, Kanematsu T. Expression of the Ets-1 proto-oncogene is linked to cell differentiation of human hepatocellular carcinoma. Hepatogastroenterology 2002;49:747-51.

16. Ozaki I, Mizuta T, Zhao G, Yotsumoto H, Hara T, Kajihara S, et al. Involvement of the Ets-1 gene in overexpression of matrilysin in human hepatocellular carcinoma. Cancer Res 2000;60:6519-25. 
17. Saeki H, Kuwano H, Kawaguchi H, Ohno S, Sugimachi K. Expression of ets-1 transcription factor is correlated with penetrating tumor progression in patients with squamous cell carcinoma of the esophagus. Cancer 2000;89:1670-6.

18. Pande P, Soni S, Kaur J, Agarwal S, Mathur M, Shukla NK, et al. Prognostic factors in betel and tobacco related oral cancer. Oral Oncol 2002;38:491-9.

19. Pande P, Soni S, Chakravarti N, Mathur M, Shukla NK, Ralhan R. Prognostic impact of Ets-1 overexpression in betel and tobacco related oral cancer. Cancer Detect Prev 2001; 25:496-501.

20. Pande P, Mathur M, Shukla NK, Ralhan R. Ets-1: a plausible marker of invasive potential and lymph node metastasis in human oral squamous cell carcinomas. J Pathol 1999;189: $40-5$.

21. Sasaki H, Yukiue H, Moiriyama S, Kobayashi Y, Nakashima Y, Kaji M, et al. Clinical significance of matrix metalloproteinase- 7 and Ets-1 gene expression in patients with lung cancer. J Surg Res 2001;101:242-7.

22. Takanami I, Takeuchi K, Karuke M. Expression of ETS-1 is correlated with urokinase-type plasminogen activator and poor prognosis in pulmonary adenocarcinoma. Tumour Biol 2001;22:205-10.

23. Nakayama T, Ito M, Ohtsuru A, Naito S, Nakashima M, Sekine I. Expression of the ets-1 proto-oncogene in human thyroid tumor. Mod Pathol 1999;12:61-8.

24. de Nigris F, Mega T, Berger N, Barone MV, Santoro M, Viglietto G, et al. Induction of ETS-1 and ETS-2 transcription factors is required for thyroid cell transformation. Cancer Res 2001;61:2267-75.

25. Davidson B, Risberg B, Goldberg I, Nesland JM, Berner A, Trope CG, et al. Ets-1 mRNA expression in effusions of serous ovarian carcinoma patients is a marker of poor outcome. Am J Surg Pathol 2002;26:539.

26. Davidson B, Reich R, Goldberg I, Gotlieb WH, Kopolovic J, Berner A, et al. Ets-1 messenger RNA expression is a novel marker of poor survival in ovarian carcinoma. Clin Cancer Res 2001;7:551-7.

27. Kitange G, Tsunoda K, Anda T, Nakamura S, Yasunaga A, Naito S, et al. Immunohistochemical expression of Ets-1 transcription factor and the urokinase-type plasminogen activator is correlated with the malignant and invasive potential in meningiomas. Cancer 2000;89:2292-300.

28. Kitange G, Kishikawa M, Nakayama T, Naito S, Iseki M, Shibata S. Expression of the Ets-1 proto-oncogene correlates with malignant potential in human astrocytic tumors. Mod Pathol 1999;12:618-26.

29. Naito S, Shimizu K, Nakashima M, Nakayama T, Ito T, Ito M, et al. Overexpression of Ets-1 transcription factor in angiosarcoma of the skin. Pathol Res Pract 2000;196:103-9.
30. Gershtein ES, Medvedeva SV, Babkina IV, Kushlinskii NE, Trapeznikov NN. Tissue- and urokinase-type plasminogen activators and type 1 plasminogen activator inhibitor in melanomas and benign skin pigment neoplasms. Bull Exp Biol Med 2001;132:670-4.

31. Nikkola J, Vihinen P, Vlaykova T, Hahka-Kemppinen M, Kahari VM, Pyrhonen S. High expression levels of collagenase-1 and stromelysin-1 correlate with shorter disease-free survival in human metastatic melanoma. Int J Cancer 2002;97: 432-8.

32. Maroulakou IG, Papas TS, Green JE. Differential expression of ets-1 and ets-2 proto-oncogenes during murine embryogenesis. Oncogene 1994;9:1551-65.

33. Dhulipal PD. Ets oncogene family. Indian J Exp Biol 1997; 35:315-22.

34. Rutter JL, Mitchell TI, Buttice G, Meyers J, Gusella JF, Ozelius LJ, et al. A single nucleotide polymorphism in the matrix metalloproteinase-1 promoter creates an Ets binding site and augments transcription. Cancer Res 1998;58:5321-5.

35. Wandel E, Grasshoff A, Mittag M, Haustein UF, Saalbach A. Fibroblasts surrounding melanoma express elevated levels of matrix metalloproteinase-1 (MMP-1) and intercellular adhesion molecule-1 (ICAM-1) in vitro. Exp Dermatol 2000;9: 34-41.

36. Barnhill RL, Fandrey K, Levy MA, Mihm MC Jr, Hyman B. Angiogenesis and tumor progression of melanoma. Quantification of vascularity in melanocytic nevi and cutaneous malignant melanoma. Lab Invest 1992;67:331-7.

37. Erhard H, Rietveld FJ, van Altena MC, Brocker EB, Ruiter DJ, de Waal RM. Transition of horizontal to vertical growth phase melanoma is accompanied by induction of endothelial growth factor expression and angiogenesis. Melanoma Res 1997;7 (Suppl 2):S19-26.

38. Graham CH, Rivers J, Kerbel RS, Stankiewicz KS, White WL. Extent of vascularization as a prognostic indicator in thin $(<0.76 \mathrm{~mm})$ malignant melanomas. Am J Pathol 1994;145: $510-4$.

39. Rongioletti F, Miracco C, Gambini C, Pastorino A, Tosi P, Rebora A. Tumor vascularity as a prognostic indicator in intermediate thickness (0.76-4.00 mm thick) cutaneous melanoma. A quantitative assay. Am J Dermatopathol 1996; 18:474-7.

40. Vlaykova T, Muhonen T, Hahka-Kemppinen M, Pyrhonen S, Jekunen A. Vascularity and prognosis of metastatic melanoma. Int J Cancer 1997;74:326-9.

41. Hudson AR, Smoller BR. Immunohistochemistry in diagnostic dermatopathology. Dermatol Clin 1999;17:667-89.

42. Schach CP, Smoller BR, Hudson AR, Horn TD. Immunohistochemical stains in dermatopathology. J Am Acad Dermatol 2000;43:1094-100. 